\title{
Data Replication Techniques for Data-Intensive Applications
}

\author{
Jaechun $\mathrm{No}^{1}$, Chang Won $\mathrm{Park}^{2}$, and Sung Soon Park ${ }^{3}$ \\ 1 Dept. of Computer Software, \\ College of Electronics and Information Engineering, \\ Sejong University, Seoul, Korea \\ 2 Intelligent IT System Research Center, \\ Korea Electronics Technology Institute, \\ Bundang-gu, Seongnam-si, Korea \\ 3 Dept. of Computer Science \& Engineering, \\ College of Science and Engineering, \\ Anyang University, Anyang, Korea
}

\begin{abstract}
Several data replication techniques have been developed to support high-performance data accesses to the remotely produced scientific data. Most of those techniques, however, do not provide the replica consistency because the data replica is just periodically updated through the remote clients. We have developed two kinds of data replication techniques, called owner-initiated replication and client-initiated replication. Our replication techniques do not need to use file system-level locking functions so that they can easily be ported to any of file systems. In this paper we describe the design and implementation of our two replication techniques and present performance results on Linux clusters.
\end{abstract}

\section{Introduction}

Many large-scale scientific experiments and simulations generate very large amounts of data [1,2, 3] (on the order of several hundred gigabytes to terabytes) in the geographically distributed storages. These data are shared between the researchers and colleagues for data analysis, data visualization, and so forth.

In order to reduce the communication and $\mathrm{I} / \mathrm{O}$ cost, the remotely located users replicate the data sets needed for their research in the local storage. The usual way of replicating data sets is to periodically update the remotely located data replicas, like implemented in Globus toolkit [4,5]. This method, however, does not provide the replica consistency in such a case that a data replica stored in more than one remote site is modified by a remote client and accessed by another client located at the other remote site before the modification to the replica is applied to the remote locations.

In order to provide the data replica consistency, we have developed two kinds of data replication techniques, called owner-initiated replication and clientinitiated replication. We integrated those replication techniques to GEDAS (Grid 
Environment-based Data Management System ) [6, 7] that is a grid toolkit providing a high-level, user-friendly interface to share the remotely produced data among the grid communities.

In the owner-initiated replication, the data owner who owns the application data sets starts the data replication to share the data sets with remote clients. In the client-initiated replication, the client who needs the data sets starts the data replication. Moreover, our replication techniques do not need to use file systemlevel locking functions so that they can easily be ported to any of file systems.

The rest of this paper is organized as follows. In Section 2, we discuss an overview of GEDAS architecture to integrate our replication techniques with GEDAS. In Section [3, we present the design and implementation of our two kinds of replication techniques. Performance results on the Linux cluster located at Sejong University are presented in Section 4. We conclude in Section 5.

\section{GEDAS Architecture}

\subsection{Overview of GEDAS}

In GEDAS, users on the data owner run large-scale, data-intensive applications while writing large amounts of data to the local storage. The remote clients who want to share the application data sets with the data owner for the purpose of data visualization or data analysis are grouped into several client groups, according to the data sets replicated on the local storage. In other words, the remote clients sharing the same replicas belong to the same client group.

Figure 1shows an overview of GEDAS. The six metadata database tables and the application data sets generated by users are located at the data owner and $n$

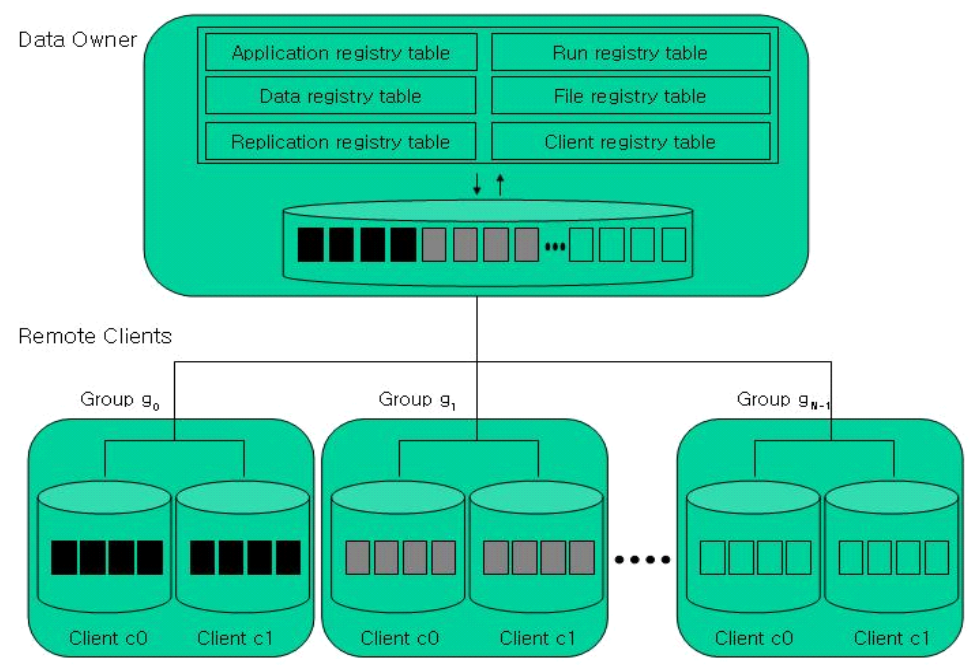

Fig. 1. An Overview of GEDAS 
remote client groups are created based on the data replicas shared among them. Each client in a group is identified with groupID and clientID, such as $\left(g_{0}, c_{0}\right)$ for the first client and $\left(g_{0}, c_{1}\right)$ for the second client in Group $g_{0}$.

The reason for making the client groups is that if a client modifies the data replicas stored in its local storage, GEDAS can easily detect the other clients who share the same data replicas and can let them take the new copy of the modified data, without affecting other clients.

\section{Data Replication}

\subsection{Owner-Initiated Data Replication}

In order to maintain the replica consistency among the remote clients, we developed two replication approaches, called owner-initiated replication and clientinitiated replication.

In the owner-initiated replication, when user generates data sets on the data owner, GEDAS replicates them to the remote clients who registered to GEDAS to share the data sets with the data owner. When a remote client changes the data replicas stored in its local storage, it broadcasts the modifications to the members in the same group where it belongs to and to the data owner for replica consistency. Figure 2 shows the steps taken in the owner-initiated replication.

Suppose that a client belonging to Group $g_{0}$ modifies the data replicas at time $t_{i}$. The client first sends the request for the IP address of other clients in $g_{0}$ to the data owner. After the client receives the IP address, it sends the modified data to the other clients in $g_{0}$ and to the data owner and waits for the acknowledgement.

When the data owner receives the modified data, it updates them to the local storage and sets the status field of the replication_registry_table to "holding"

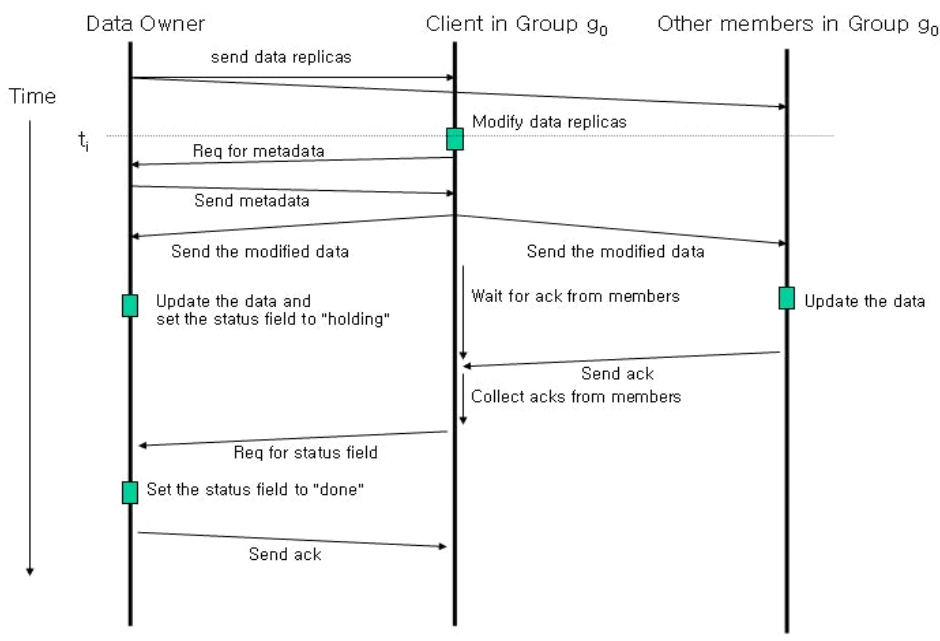

Fig. 2. Owner-Initiated Replication 
to prevent another client from accessing the data sets while being updated. When the data owner receives the signal from the client who initiated the data modification, it sets the status field to "done", allowing another client to use the data replica.

The owner-initiated replication approach allows remote clients to share the data replicas safely, provided that they find out the corresponding status field is set to "done". Moreover, a remote client crash doesn't affect to the data consistency because as soon as the data replicas are modified the change is immediately reflected to the data owner and the other clients in the same group. However, if the data modification to the data replicas frequently happens, the heavy communication bottleneck then incurs even if no one else would use the data sets modified.

\subsection{Client-Initiated Data Replication}

Figure 3 shows the client-initiated replication where only when the modified data replicas are needed by users are those data replicas sent to the requesting client and to the data owner. Unlike in the owner-initiated replication, there is no data communication when users on the data owner produce the application data sets. If a client needs to access the remote data sets stored in the data owner, he will then get the data replica while registering to GEDAS.

Suppose that client A belonging to Group $g_{0}$ modifies a data replica at time $t_{i}$. He just sends a signal to the data owner to update the corresponding status field of the data set to the IP address of client A.

At time $t_{j}$, let client $\mathrm{B}$ access the data replica stored in its local storage but not been updated by client A's data modification. In order to check the replica

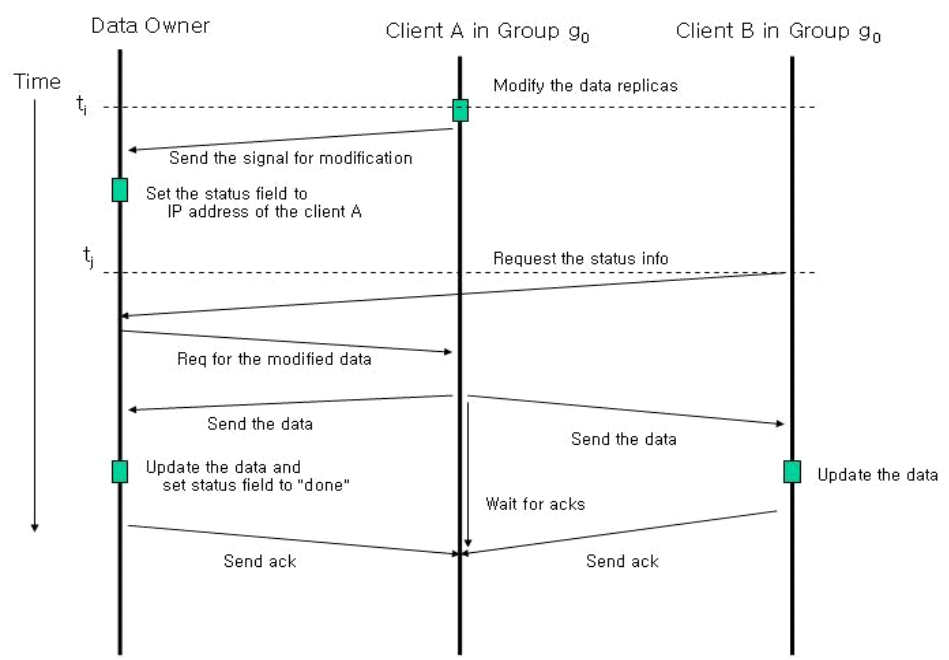

Fig. 3. Client-Initiated Replication 
consistency, client B first requests the status information of the data replica to the data owner. The data owner finds out that the data set has been modified by client $\mathrm{A}$ and requests the data to client $\mathrm{A}$. Client $\mathrm{A}$ sends the modified data to the data owner and to the client $\mathrm{B}$, and then waits for the acknowledgement from both. After the data owner updates the modified data set and sets the status field to "done", it sends back an acknowledgement to the client A.

In the client-initiated replication approach, the data replicas are sent to the remote clients only when the data sets are actually needed by them. Therefore, unlike in the owner-initiated replication approach, the client-initiated replication does not incur unnecessary data communication. However, if a client who keeps the modification of the data replica is crashed before the data modification is updated to the data owner and to the other members of the same group, a significant data loss will be happened.

\section{Performance Evaluation}

In order to measure the performance, we used two Linux clusters located at Sejong university. Each cluster consists of eight nodes having Pentium $3866 \mathrm{MHz}$ CPU, $256 \mathrm{MB}$ of RAM, and 100Mbps of Fast Ethernet each. The operating system installed on those machines was RedHat 9.0 with Linux kernel 2.4.20-8.

The performance results were obtained using the template implemented based on the three-dimensional astrophysics application, developed at the University of Chicago. The total data size generated was about 520MB and among them, $400 \mathrm{MB}$ of data were generated for data analysis and data restart, and then the remaining $120 \mathrm{MB}$ of data were generated for data visualization. The data sets produced for visualization are used by the remote clients, thus requiring the data replication to minimize the data access time.

In order to evaluate two replication approaches, a randomly chosen remote client modified $30 \mathrm{MB}$ of replicas at time steps 5,10 , and 15 and spread those replicas to the data owner and to the clients, according to the owner-initiated replication and to the client-initiated replication. A maximum execution time for the data replication measured among the remote clients was selected as a performance result. This time includes the cost for metadata access on the data owner, real data communication, and I/O operations.

In Figure 4, we made two client groups, while each group consisting of two nodes. At each time step, a client accesses either $30 \mathrm{MB}$ of replicas stored in the local storage, or $30 \mathrm{MB}$ of remote data sets stored on the data owner in such a case that the data sets required are not replicated.

In the owner-initiated replication, as soon as an application produces data sets at time step 0 , all the remote clients receive the necessary visualization data sets to replicate them to the local storage. These replicas are used until the modification to the replicas happens at time steps 5, 10, and 15 , respectively.

When the replicas stored in the local storage are used, the execution time for accessing visualization data sets drops to almost about 3 seconds needed for communicating the corresponding status information with the data owner. 


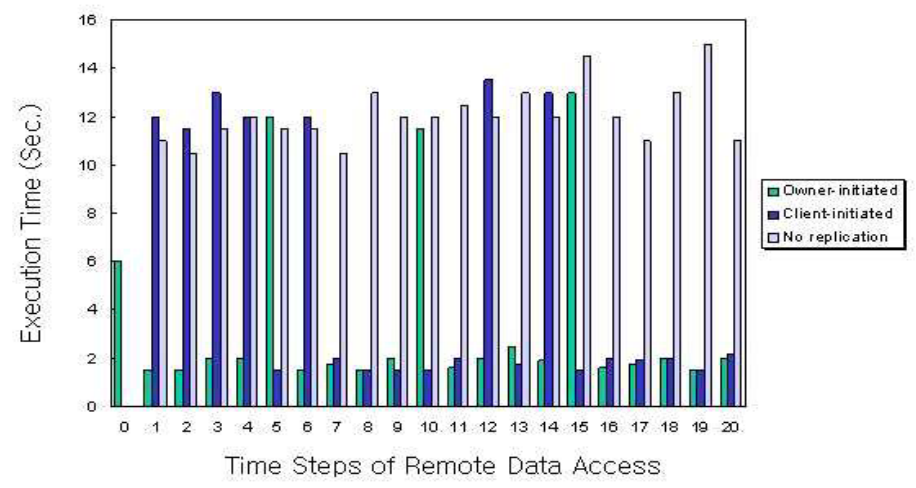

Fig. 4. Execution time for replicating visualization data on the remote clients as a function of time steps for accessing remote data sets. Two client groups were made, while each group consisting of two nodes.

If the modification to the replicas happens, like occurred at time steps 5,10 , and 15 , respectively, the modified replicas are then broadcast to the data owner and to the clients in the sample group, thereby increasing the execution time for accessing remote data sets.

In the client-initiated replication, since there is no replica stored in the client side until time step 4, each remote client should communicate with the data owner to receive the data sets needed. Because each client can use the data replicas stored in its local storage from time step 5, the execution time for accessing data sets dramatically drops to almost 3 seconds.

When the replicas are modified at time steps 5,10 , and 15 , the client-initiated approach just sends the IP address of the client modifying the replicas, and thus

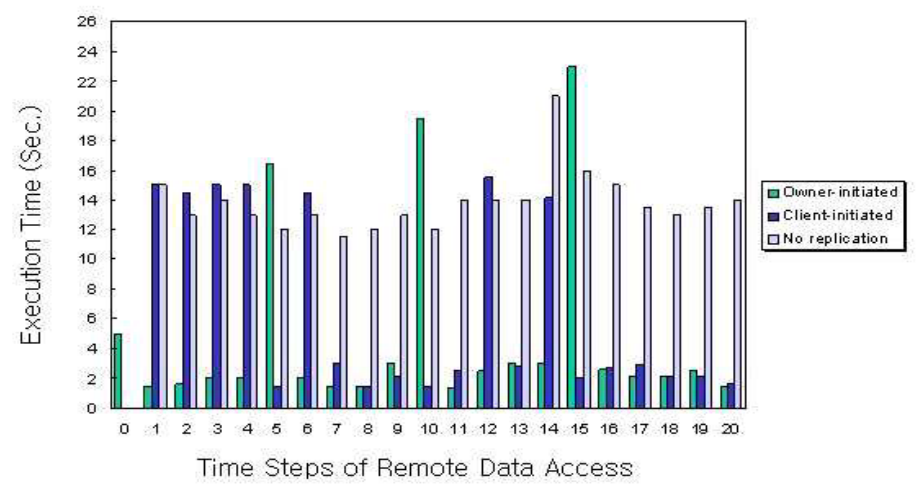

Fig. 5. Execution time for replicating visualization data on the remote clients as a function of time steps for accessing remote data sets. Two client groups were made, while each group consisting of eight nodes. 
it takes no more than 3 seconds. However, we can see that in Figure 4 at time steps 6,12 , and 14, another client tries to access the modified replicas, thus occurring the data communication and I/O cost to update the replicas to the requesting client and to the data owner.

Without data replication, the data communication for accessing the remote data sets consistently happens on the clients, affecting the performance.

In Figure 5. we increased the number of nodes in each group to eight. With this configuration, when the replicas are modified at a remote client, the execution time for accessing data sets in the owner-initiated replication is significantly increased, compared to Figure 4, because of the increment in the communication cost to broadcast the replicas to the data owner and to the other clients.

On the other hand, as can be seen in Figure [5] the client-initiated replication shows not much difference in the execution time to receive the modified data sets because less number of nodes than in the own-initiated replication is involved in the communication. However, we believe that more performance evaluations should be conducted to present some valuable conclusions with these two replication approaches.

\section{Conclusion}

We have developed two data replication approaches to maintain the replica consistency in case of replica modifications or updates. In the owner-initiated replication, the replication occurs when applications generate the data sets in the data owner location. Whenever a remote client modifies or updates its replica, in order to maintain the data consistency, it broadcasts the replica to the other members of the same group, as well as to the data owner retaining the entire application data sets. In the client-initiated replication, only when the data sets are needed by a remote client are the necessary data sets replicated on the requesting client. If a client modifies its replica, it just sends the signal to the data owner in order for the other client to recognize the recently modified replica. Due to the data broadcast, the owner-approach shows the increased communication overhead when the number of nodes in a group becomes large. On the other hand, the client-initiated replication shows the constant communication cost even with the increased number of nodes in a group. In the future, we plan to use our replication techniques with more applications and evaluate both the usability and performance.

\section{References}

1. B. Allcock, I. Foster, V. Nefedova, A. Chervenak, E. Deelman, C. Kesselman, J. Leigh, A. Sim, A. Shoshani, B. Drach, and D. Williams. High-Performance Remote Access to Climate Simulation Data: A Challenge Problem for Data Grid Technologies. SC2001, November 2001

2. R. Moore, A. Rajasekar. Data and Metadata Collections for Scientific Applications. High Performance Computing and Networking (HPCN 2001), Amsterdam, NL, June 2001 
3. A. Chervenak, E. Deelman, C. Kesselman, L. Pearlman, and G. Singh. A Metadata Catalog Service for Data Intensive Applications. GriPhyN technical report, 2002

4. I. Foster, C. Kesselman, J. Nick, and S. Tuecke. The Physiology of the Grid: An Open Grid Services Architecture for Distributed Systems Integration WG, Global Grid Forum, June 22, 2002

5. A. Chervenak, I. Foster, C. Kesselman, C. Salisbury, S. Tuecke. The Data Grid: Towards an Architecture for the Distributed Management and Analysis of Large Scientific Datasets. Journal of Network and Computer Applications, 23:187-200, 2001

6. J. No, H. Park. GEDAS: A Data Management System for Data Grid Environments. In Proceedings of International Conference on Computational Science, 2005, pages 485-492

7. J. No, R. Thakur, and A. Choudhary. High-Performance Scientific Data Management System. Journal of Parallel and Distributed Computing, (64)4:434-447, April 2003 